\title{
NANOTECNOLOGIA E O MEIO AMBIENTE: PERSPECTIVAS E RISCOS
}

\begin{abstract}
A editoria de Química Nova recebeu esta carta do Prof. Frank Quina, um dos diretores do CEPEMA (USP), onde é exposta uma reflexão sobre o desenvolvimento da nanotecnologia e seu impacto sobre o meio ambiente. A matéria é considerada de alta prioridade científica e merece a análise e reflexão de toda a comunidade Química.
\end{abstract}

A nanotecnologia estende a ciência de materiais para o domínio de partículas e interfaces com dimensões extremamente pequenas, da ordem de um a cem nanômetros. Partículas deste tamanho, ou "nanopartículas", apresentam uma grande área superficial e, freqüentemente, exibem propriedades mecânicas, ópticas, magnéticas ou químicas distintas de partículas e superfícies macroscópicas. $\mathrm{O}$ aproveitamento dessas propriedades em aplicações tecnológicas forma a base da nanotecnologia de materiais. Há, também, uma área ainda incipiente da nanotecnologia, denominada nanotecnologia molecular ou nano-fabricação ("nanomanufacturing"), que almeja o desenvolvimento de sistemas nanométricos auto-replicantes (nano-robôs ou "nano-bots") capazes de fabricar, sob medida, materiais ou objetos através da manipulação da matéria a nível molecular.

Não há dúvida de que a nanotecnologia oferece a perspectiva de grandes avanços que permitam melhorar a qualidade de vida e ajudar a preservar o meio ambiente. Entretanto, como qualquer área da tecnologia que faz uso intensivo de novos materiais e substâncias químicas, ela traz consigo alguns riscos ao meio ambiente e à saúde humana. Nos próximos parágrafos, analisaremos resumidamente os possíveis benefícios e perigos da nanotecnologia.

As três principais áreas nas quais podemos esperar grandes benefícios provenientes da nanotecnologia são $\mathrm{O}^{1-3}$ :

(a) na prevenção de poluição ou dos danos indiretos ao meio ambiente. Por exemplo, o uso de nanomateriais catalíticos que aumentam a eficiência e a seletividade de processos industriais resultaria num aproveitamento mais eficiente de matérias primas, com consumo menor de energia e produção de quantidades menores de resíduos indesejáveis. A nanotecnologia vem contribuindo para o desenvolvimento de sistemas de iluminação de baixo consumo energético. Naárea da informática, o uso de nanoestruturas de origem biológica pode oferecer uma estratégia alternativa para a fabricação de dispositivos microeletrônicos. A nanotecnologia também vem aprimorando o desenvolvimento de displays (como, por exemplo, monitores de computador ou displays dobráveis de plástico que podem ser lidos como uma folha de papel) que, além de serem mais leves e possuirem melhor definição, apresentam as vantagens da ausência de metais tóxicos na sua fabricação e de terem um consumo menor de energia.

(b) no tratamento ou remediação de poluição. A grande área superficial das nanopartículas lhes confere, em muitos casos, excelentes propriedades de adsorção de metais e substâncias orgânicas. A etapa subseqüente de coleta das partículas e remoção de poluentes pode ser facilitada pelo uso, por exemplo, de nanopartículas magnéticas. As propriedades redox e/ou de semicondutor de nanopartículas podem ser aproveitadas em processos de tratamento de efluentes industriais e de águas e solos contaminados baseados na degradação química ou fotoquímica de poluentes orgânicos. Num cenário futurístico, um exército de nano-bots poderia ser utilizado para descontaminar microscopicamente sítios de derrame de produtos químicos.

(c) na detecção e monitoramento de poluição. A nanotecnologia vem permitindo a fabricação de sensores cada vez menores, mais seletivos e mais sensíveis para a detecção e monitoramento de poluentes orgânicos e inorgânicos no meio ambiente. Avanços em sensores para a detecção de poluentes implicam diretamente num melhor controle de processos industriais; na detecção mais precoce e precisa da existência de problemas de contaminação; no acompanhamento, em tempo real, do progresso dos procedimentos de tratamento e remediação de poluentes; num monitoramento mais efetivo dos níveis de poluentes em alimentos e outros produtos de consumo humano; na capacidade técnica de implementar normas ambientais mais rígidas, etc.

Não obstante estas perspectivas animadoras dos benefícios da nanotecnologia para a melhoria do meio ambiente, não se deve subestimar o potencial para danos ao meio ambiente ${ }^{2-10}$. As mesmas características que tornam as nanopartículas interessantes do ponto de vista de aplicação tecnológica, podem ser indesejáveis quando essas são liberadas ao meio ambiente. O pequeno tamanho das nanopartículas facilita sua difusão e transporte na atmosfera, em águas e em solos, ao passo que dificulta sua remoção por técnicas usuais de filtração. Pode facilitar também a entrada e o acúmulo de nanopartículas em células vivas. De modo geral, sabe-se muito pouco ou nada sobre a biodisponibilidade, biodegradabilidade e toxicidade de novos nanomateriais. A contaminação do meio ambiente por nanomateriais com grande área superficial, boa resistência mecânica e atividade catalítica pode resultar na concentração de compostos tóxicos na superfície das nanopartículas, com posterior transporte no meio ambiente ou acúmulo ao longo da cadeia alimentar; na adsorção de biomoléculas, com conseqüente interferência em processos biológicos in vivo; numa maior resistência à degradação (portanto, maior persistência no meio ambiente) e em catálise de reações químicas indesejáveis no meio ambiente.

Apesar da crescente preocupação em relação às potencialidades negativas da nanotecnologia para o meio ambiente e/ou para a saúde humana, ela ainda não enfrenta nenhuma oposição tecnofóbica sistemática (algumas exceções isoladas incluem um pedido de moratória na pesquisa em nanotecnologia molecular, feito pelo ETC Group ${ }^{12}$ do Canadá, e uma obra de ficção sobre as consequiências de liberação de nano-bots auto-replicantes ao meio ambiente ${ }^{13}$ ). Aliás, quase todos os simpósios e estudos recentes ${ }^{2,4-11}$ que avaliaram os riscos da nanotecnologia para o meio ambiente tratam a questão com equilíbrio e em termos predominantemente científicos. Três desses estudos, todos disponíveis pela Internet, merecem destaque:

(1) A. H. Arnall, "Future Technologies, Today's Choices: Nanotechnology, Artificial Intelligence and Robotics; A technical, political and institutional map of emerging technologies"8, de 2003, da conhecida organização ecológica Greenpeace Environmental Trust, que trata de forma bastante equilibrada a questão;

(2) "Nanotechnology: small matter, many unknowns", , de 2004, da resseguradora internacional Swiss Re, que alerta para o estado de desconhecimento dos riscos associados à nanotecnologia $\mathrm{e}$ enfatiza a necessidade de normas e pesquisa que delineiem e minimizem esses riscos para as companhias de seguros;

(3) "Nanoscience and nanotechnologies: opportunities and uncertainties" 10 , da Academia Real de Engenharia e da Sociedade Real do UK, de julho de 2004, que recomenda, entre outras coisas, cuidado na manipulação ou aplicação de nanopartículas artificiais em forma livre (e.g., em processos voltados para a remediação do meio ambiente) e o tratamento de nanopartículas e nanotubos como substâncias químicas novas para fins regulatórios. Aponta ainda a 
necessidade de pesquisa interdisciplinar voltada para as questões de toxicidade, epidemiologia, persistência e bioacumulação de nanopartículas.

Evidentemente, cabe à nossa comunidade avaliar continuamente as tecnologias em desenvolvimento nos laboratórios do ponto de vista do seu potencial de risco, buscando conscientemente soluções e alternativas que eliminem ou minimizem os possíveis danos ao meio ambiente ou à saúde, principalmente daqueles que manipulam nanopartículas em nossos laboratórios. Neste contexto, são da mais alta relevância iniciativas como o Edital MCT/CNPq 13/2004, vinculado ao Programa de Desenvolvimento da Nanociência e da Nanotecnologia, que contemplou "apoio a atividades de pesquisa voltadas para o estudo de aspectos éticos ou impactos ambientais da nanotecnologia e nanobiotecnologia".

Frank H. Quina Instituto de Química, Universidade de São Paulo, CP 26077, 05513-970 São Paulo - SP e Centro de Capacitação e Pesquisa em Meio Ambiente (CEPEMA-USP), Depto. de Engenharia Química,

Escola Politécnica, USP, Cubatão - SP (em fase de implantação) e-mail: quina@usp.br

\section{REFERÊNCIAS}

1. U. S. Environmental Protection Agency (EPA), STAR Progress Review Workshop, "Nanotechnology and the Environment: Applications and Implications", 2002: http://es.epa.gov/ncer/publications/workshop/ nano_proceed.pdf, acessada em setembro, 2004.
2. Masciangioli, T.; Zhang, W.-T. Environ. Sci. Technol. 2003, 37, 102A.

3. A. Keiper, The New Atlantis, 2003, No. 2 (Summer), p. 17-34: http:// www.thenewatlantis.com/archive/2/keiperprint.htm, acessada em setembro, 2004.

4. Potential Risks of Nanotechnologies on the Environment and the Food Chain: www.azonano.com/details.asp?ArticleID $=1007$, acessada em setembro, 2004.

5. Oberdörster, E.; Environ. Health Perspect. 2004, 112, 1058.

6. Feder, T. Physics Today 2004, 57, 30.

7. Dagani, R. "Nanomaterials: Safe or Unsafe?", Chem. Eng. News 2003, 81, 30.

8. http://www.greenpeace.org.uk/MultimediaFiles/Live/FullReport/5886.pdf, acessada em setembro, 2004.

9. http://www.swissre.com/INTERNET/pwsfilpr.nsf/vwFilebyIDKEYLu/ ULUR-5YNGET/\$FILE/Publ04_Nanotech_en.pdf, acessada em setembro, 2004.

10. http://www.nanotec.org.uk/finalReport.htm, acessada em setembro , 2004. Um estudo semelhante da Academia Nacional de Ciências dos EUA deve ser concluído em 2005. O relatório do UK descarta como improvável uma das nanofobias mais populares, a catástrofe "gray goo", em que uma gosma cinza formada pela multiplicação desenfreada de nanorobôs auto-replicantes cresce até o ponto de asfixiar a vida do planeta.

11. Mais informação está disponível nos Websites de organizações como: o Center for Responsible Nanotechnology (http://crnano.org): o Center for Biological and Environmental Nanotechnology (CBEN) (http:// www.ruf.rice.edu/ cben/), associado ao Environmental \& Energy Systems Institute da Rice University (http://eesi.rice.edu/nanotech.cfm), mantido pela Fundação Nacional de Ciência (NSF) dos EUA; e o Foresight Institute (http://www.foresight.org/).

12. Action Group on Erosion, Technology and Concentration: http:// www.etcgroup.org/search.asp?theme=11, acessada em setembro, 2004. Vide também: Águas Turbulentas da Nanotecnologia ("Nano's Troubled Waters") http://www.etcgroup.org/documents/GT_TroubledWater_April1.pdf, acessada em setembro, 2004.

13. Crichton, M.; Prey, HarperCollins Publishers: New York, 2002; publicado no Brasil com o título Presa, Editora Rocco: São Paulo, 2003. 